\title{
ESPECIES DE ALTERNARIA DE LA SABANA DE BOGOTÁ, COLOMBIA
}

Fecha de recepción: 4 de julio de 2013 • Fecha de aceptación: 19 de agosto de 2013

\section{ALTERNARIA SPECIES IN THE BOGOTÁ PLATEAU, COLOMBIA}

Rodríguez-Roa, John Henry $\bullet$ Cárdenas, Martha E. ${ }^{2} \bullet J^{1}$ Jiménez, Pedro ${ }^{3^{\star}}$

\section{RESUMEN}

El género Alternaria contiene algunos de los patógenos de plantas más importantes en el mundo. En Colombia existen reportes de enfermedades producidas por Alternaria en cultivos de importancia como solanáceas y cítricos, sin embargo, frecuentemente los diagnósticos no incluyen la determinación de las especies que las producen. Este estudio se realizó con el fin de incrementar el conocimiento de la diversidad del género Alternaria en Colombia. Muestras vegetales que presentaban lesiones características producidas por el patógeno fueron colectadas de diferentes sitios de la sabana de Bogotá. Después de ser desinfectadas, las muestras fueron cultivadas en Agar Papa-Dextrosa (PDA). La determinación taxonómica de los aislamientos se realizó de acuerdo a las descripciones de Simmons (2007). Se obtuvieron 50 aislados pertenecientes a 14 especies de Alternaria, en 16 hospederos, principalmente solanáceas y ornamentales. Todas las especies identificadas hacen parte de la sección de esporas pequeñas, siendo $A$. alternata la más frecuente. A pesar de la alta proporción de solanáceas dentro de los hospederos no se pudo determinar la presencia de $A$. solani (especie arquetípica dentro de la familia). Se realizó una caracterización morfométrica de los conidios, además de una descripción de las colonias y el patrón de esporulación, con los cuales se elaboró y se sustentó una clave taxonómica de las especies de Alternaria en la sabana de Bogotá, que se acompaña de descripciones e imágenes de referencia.

Palabras clave: Alternaria, morfología, descripción, clave taxonómica.

\section{ABSTRACT}

The genus Alternaria contains some of the major plant pathogens in the world. In Colombia Alternaria has been reported attacking important crops like citrus, ornamentals, and the Solanaceae family. However, frequently, the diagnostic does not include determining the species. This study was performed to increase our knowledge of the diversity of this genus in Colombia. Plant samples with characteristic lesions produced by the pathogen, from different sites in the Bogota plateau, were collected and taken to the laboratory. Samples were

1. Maestría en Biología Aplicada, Universidad Militar Nueva Granada

2. Laboratorio de Micología y Fitopatología, Universidad de Los Andes, Bogotá, Colombia.

3. Laboratorio de Fitopatología, Universidad Militar Nueva Granada, Campus Nueva Granada, Km 2 vía Cajicá-Zipaquirá, Cundinamarca, Colombia.

* Autor para correspondencia: pedro.jiménez@unimilitar.edu.co 
then surface desinfected, and cultured on Potato Dextrose Agar (PDA). The taxonomical determination of the isolates was done following the descriptions in Simmons (2007). From 16 plant hosts, mainly Solanaceae and ornamentals, fifty isolates were recovered, and 14 Alternaria species were identified. All the recovered species belonged to the small spores section. The most common isolated species was A. alternata, and occurrence of A. solani, archetypal species for the Solanaceae, was not recorded despite the high frequency of this botanical family among the plants collected. We performed a morphometric characterization of conidia, and also a description of the colonies and sporulation patterns. A taxonomic key to the species of Alternaria in the Bogotá plateau descriptions, and reference images are presented.

Keywords: Alternaria, morphology, description, taxonomic key.

\section{INTRODUCCIÓN}

Alternaria, Nees, es un género de hongos, perteneciente al Filo Ascomycota. Sus especies se distribuyen globalmente, por lo que se considera un hongo cosmopolita. Se caracteriza además por presentar un gran número de roles ecológicos. Se ha discutido su papel como endófito (Aly et al., 2008; Lahlali y Hijri, 2010; Kaur et al., 2013), saprófito o parásito de plantas vivas (Pitt y Hocking, 1997). La base de datos de hongos del Departamento de Agricultura de los Estados Unidos (USDA) presenta 4000 asociaciones de Alternaria con diferentes hospederos (Farr et al., 2013), convirtiéndose en uno de los géneros de hongos con más amplio rango de hospederos como patógeno de plantas (Andrew et al., 2009). Farr et al. (1989), posicionan a Alternaria en el décimo lugar en términos del número total de plantas hospederas. Solo una especie, A. alternata es responsable de infectar más de 100 especies vegetales (Simmons, 1992; Simmons, 2007).

Las especies de Alternaria se caracterizan por producir una variedad de compuestos tóxicos, que además de determinar la patogenicidad en plantas (Ostry, 2008; Barkai-Golan et al., 2008), se han asociado con efectos adversos para la salud humana y animal, razón por la cual su estudio ha recibido una creciente atención, tanto en programas de investigación, como en estudios de evaluación de riesgo epidemiológico (Ostry, 2008).

La determinación del número de especies que constituyen el género está aún en discusión. Las estimaciones más conservadoras proponen 44 especies (Rotem, 1994). Sin embargo, revisiones más recientes exponen un número mucho mayor. Simmons (2007) acepta y describe 276 especies en su manual de identificación, mientras que Kirk et al. (2008), en su diccionario de hongos, reportan 299 especies. Finalmente, la base de datos de nomenclatura de hongos del Departamento de Agricultura de Estados Unidos (USDA), arroja un total de 418 registros dentro del género (Cline et al., 2014).

La clasificación de las especies de Alternaria se sustenta en las características morfológicas, particularmente las que se relacionan con sus conidios; el tamaño, la forma, y la existencia y estructura de sus picos han sido caracteres fundamentales para separar especies (Joly, 1964; Ellis, 1971; Ellis, 1976; Simmons, 1997). Igualmente, el patrón de esporulación, - la estructura de las cadenas de conidios han sido empleados como una herramienta clave en la taxonomía del género. Neergard (1945) propone tres grupos de especies, referidos a la longitud de las cadenas de conidios, Longicatenado (diez o más conidios), Brevicatenado (aproximadamente cinco conidios), y 
Noncatenado (conidios simples), mientras que Simmons (1993), presenta seis grupos de especies, diferenciados por la longitud de las cadenas de conidios y el tipo de ramificación que éstas presentan.

Actualmente se distinguen dos grupos de especies dentro del género, que se separan por el tamaño de sus conidios. Para la sección de conidios grandes, la naturaleza del desarrollo apical de los conidios determina la diversidad de especies, mientras que para la sección de conidios pequeños, es la relación de los conidióforos y conidios en el desarrollo del patrón de esporulación la que sustenta la existencia de varios taxones (Simmons, 2007).

La correcta identificación de algunas especies de Alternaria ofrece considerables dificultades debido a la alta variabilidad de los caracteres morfológicos de sus estructuras reproductivas (Xia y Zhang, 2008). Se estima que diversos factores tienen un efecto importante en la variabilidad de dichas características. Rotem (1994), menciona al sustrato donde crece el hongo como el factor que mayor variabilidad aporta. Simmons (1992), adiciona la temperatura y los regímenes de luz dentro de estos factores, y menciona que el manejo estricto de estos parámetros ha sido fundamental en la descripción de nuevas especies. Cuando Alternaria crece en cultivos ricos en nutrientes, medios no estandarizados, y en la oscuridad bajo condiciones ambientales no controladas, la formación de micelio aéreo en exceso evita el desarrollo de los patrones de esporulación tridimensionales característicos (Andersen et al., 2001). Un factor adicional que conduce a errores en la identificación de especies, es el uso de montajes húmedos en la descripción de los patrones de esporulación, porque éstos alteran la estructura de las cadenas, dejando como único carácter disponible, la dimensión de los conidios, lo que agrava aún más el error (Andersen et al., 2001).

A través de características morfológicas es posible distinguir entre los dos grupos principales de
Alternaria (conidios pequeños y conidios grandes), de la misma manera que es posible hacerlo para las especies que integran el grupo de conidios grandes (Pryor y Gilbertson, 2000; Chou y Wu, 2002; Pryor y Bigelow, 2003). Sin embargo la distinción dentro del grupo de conidios pequeños, especialmente las que forman cadenas, también denominada sección alternata, representa un reto taxonómico no resuelto (Sun y Zhang, 2008).

Un ejemplo concreto de estas dificultades es evidente en la posición de los investigadores con respecto a las especies que integran el grupo alternata. Rotem (1994), ha reportado la existencia de por lo menos 11 especies oscuras dentro del grupo, y expone que muchos de los taxones de hospederos específicos, como A. mali o A. gaisen, deben ser referidos como patotipos de A. alternata. Mientras que Andrew et al. (2009), argumentan que debido a la plasticidad morfológica que presenta el grupo, es imposible diferenciar morfoespecies dentro del grupo.

Sin embargo, Simmons (2007), expone la posibilidad de realizar la identificación taxonómica del género, incluyendo el grupo alternata, apelando al manejo cuidadoso de las condiciones de cultivo. Para él, y otros autores, la principal fuente de error para conseguir una identificación taxonómica correcta, fundamentada en aspectos morfológicos, dentro de todos los grupos de Alternaria es el manejo equivocado de variables que afectan el crecimiento in vitro (Simmons y Roberts, 1993; Simmons, 1999; Roberts et al., 2000). Por esta razón los autores exponen como de vital importancia, estandarizar estas variables y los protocolos de medición de las mismas.

Actualmente la sección alternata, se trata como un complejo que incluye alrededor de 60 especies, cuya diferenciación taxonómica está determinada más por diferencias moleculares que morfológicas (Woudenberg et al., 2013).

Una precisa y correcta identificación de las especies dentro del género es necesaria, no solo debido 


\section{La correcta identificación de algunas especies de Alternaria ofrece considerables dificultades debido a la alta variabilidad de los caracteres morfológicos de sus estructuras reproductivas (Xia y Zhang, 2008).}

al deseo de clasificarlas y controlarlas, sino también porque el nombre de las especies representa un conjunto de caracteres, por ejemplo, preferencias de crecimiento, patogenicidad o producción de micotoxinas, que nos permiten predecir su comportamiento (Andersen et al., 2001). Una identificación morfológica razonable de las especies de Alternaria debe depender de una combinación de las características de conidios a partir de muestras naturales, cultivos en medios artificiales y sus patrones de esporulación tridimensionales (Zhang, 2003).

El estudio de Alternaria en Colombia se ha restringido principalmente al impacto que el género tiene como patógeno, sobre cultivos específicos y de interés comercial, como algunas solanáceas (Zubieta et al., 1974; Barreto et al., 2002; Zapata et al., 2002), y cítricos (Castro et al., 2000). La base de datos de USDA reporta para Colombia Alternaria brassicae en Brassica oleracea var. capitata, A. colombiana en naranja tangelo (Citrus ${ }^{x}$ paradisireticulata), A. obclavoidea en Passiflora sp., A. porri en cebolla (Allium cepa y A. porrum), A. solani en pimentón (Capsicum annuum), tomate (Solanum lycopersicon) y papa (S. tuberosum y S. tuberosum subsp. andigenum) y A. tenuissima en andropogon (Andropogon gayanus). Más allá de estos estudios, no existe una revisión concreta de las especies de
Alternaria en alguna región de país, ni sobre la taxonomía del género.

Este estudio se plantea con el objetivo de incrementar el conocimiento de la diversidad de especies dentro del género Alternaria en la sabana de Bogotá, apelando a un enfoque morfológico, además de intentar determinar qué variables morfológicas son fundamentales para diferenciar sus especies.

\section{MATERIALES Y MÉTODOS}

\section{Colección de aislados}

En Colombia existen pocos aislados de Alternaria mantenidos en colecciones, por lo cual se realizó una colecta en algunos municipios de la sabana de Bogotá. Un total de 50 aislados pertenecientes al género, o a géneros relacionados, fueron obtenidos de plantas que presentaban síntomas característicos (Agrios, 2005). La identidad, el grupo al que corresponden de acuerdo al patrón de esporulación, el origen geográfico y el hospedero fueron registrados en la tabla 1.

Para el aislamiento, pequeñas piezas de tejido (comúnmente hoja) fueron removidas del borde de las lesiones y se desinfectaron con etanol al $70 \%$ por 30 segundos, seguido por hipoclorito al $1 \%$ por 5 minutos, después de lo cual se lavaron tres veces con agua destilada estéril. Las muestras fueron dispuestas en 
cajas de Petri con Agar Papa-Dextrosa (PDA) e incubadas a $25^{\circ} \mathrm{C}$ por 5 días. Para obtener cultivos monospóricos, se inocularon conidios en cajas de Petri con Agar Agua (AA) utilizado la técnica de siembra por agot amiento con asa microbiológica, se incubaron a $25^{\circ} \mathrm{C}$ por una noche y se recuperó un conidio germinado por aislamiento, bajo estereoscopio. Este conidio fue sembrado, y crecido, sobre un disco de papel de filtro estéril colocado sobre PDA (Oxoid) en una caja de Petri de $90 \mathrm{~mm}$ de diámetro. Una vez el hongo colonizó la superficie hasta alcanzar los bordes de la caja de Petri, el disco de papel de filtro fue retirado y colocado en una caja de Petri vacía, donde se le dejó tapado hasta su desecación total. Este disco ya deshidratado fue cortado en cuadros de $9 \mathrm{~mm}^{2}$, antes de ser introducidos en tubos de vidrio con tapa de rosca estériles, y almacenados a $-20{ }^{\circ} \mathrm{C}$. Todos los aislados fueron almacenados de esta manera, además se conservan como preparaciones liofilizadas en la colección de cultivos del Laboratorio de Fitopatología de la Universidad Militar Nueva Granada en Cajicá, Cundinamarca, Colombia.

\section{Caracterización morfológica}

Pequeñas secciones de papel filtro con el inóculo de cada uno de los aislados, fueron dispuestas en cajas de Petri de $90 \mathrm{~mm}$ de diámetro, con Agar Papa Zanahoria (PCA) y Agar V8 (Simmons, 2007). Con el fin de inducir la formación de los patrones de esporulación característicos, las cajas fueron incubadas bajo condiciones de luz natural, es decir en un ciclo alternante luz/oscuridad de 12/12 horas, a $25^{\circ} \mathrm{C}$, por 7 días.

\section{Características de la colonia}

El diámetro de las colonias fue medido después de 7 días de inoculado el medio. Además se tuvieron en cuenta otras características frecuentes de las colonias del género durante su descripción, como la aparición de anillos concéntricos y el crecimiento parcialmente sumergido del micelio en el medio.

\section{Formación de cadenas de conidios}

Se evaluó la estructura tridimensional de la cadena de conidios a través de su observación directa, bajo estereoscopio con una magnificación de 50X, y microscopio de luz con magnificación 40X, 100X y 400X. El patrón de esporulación típico de cada uno de los aislados fue fotografiado con cámara digital, y fue asignado a cada uno de los grupos descritos por Simmons (2007) (Tabla 1), en algunos casos, esquemas del patrón de esporulación fueron obtenidos

\section{El diámetro de las colonias fue medido después de 7 días} de inoculado el medio. Además se tuvieron en cuenta otras características frecuentes de las colonias del género durante su descripción, como la aparición de anillos concéntricos y el crecimiento parcialmente sumergido del micelio en el medio. 


\section{Clave para las especies de la sección de conidios pequeños de Alternaria de la Sabana de Bogotá}

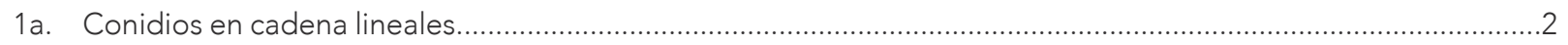

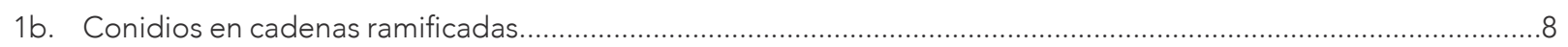

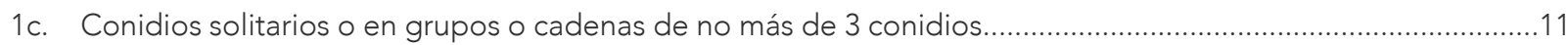

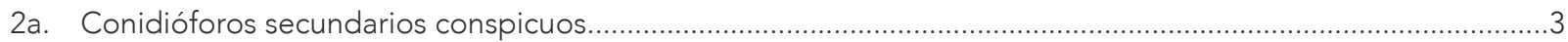

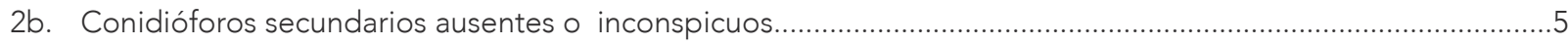

3a. Esporulación parcialmente sumergida en el medio. Conidios maduros, elipsoides a subcilíndricos, con una alta variabilidad en longitud, algunos alcanzan hasta $80 \mu \mathrm{m}$ de largo........

A. alstroemeriae

3b. Esporulación aérea. Conidios maduros cortos (hasta $30 \mu \mathrm{m}$ de largo), ovoides o elipsoides. 4

4a. Conidióforos secundarios largos (hasta $250 \mu \mathrm{m}$ ) A. astragali

4b. Conidióforos secundarios cortos (hasta $50 \mu \mathrm{m}$ ) A. colombiana

5a. Cadenas cortas (3-7 conidios menos de 12 conidios)..... 6

5b. Cadenas largas (5-15 conidios más de 12 conidios), ocasionalmente una ramificación lateral corta... A. tenuissima

6a. Conidios ovoides o elipsoides anchos.

6b. Conidios ovoides o elipsoides a ovales, 10-30 x 5-10 $\mu \mathrm{m}$. A. godetiae

7a. Hasta 8 conidios por cadena, $15-40 \times 7-20 \mu \mathrm{m}$........ A. gaisen

7b. Hasta 12 conidios por cadena, 11-50 x 7-18 $\mu \mathrm{m}$ A. pellucida

8a. Conidióforos secundarios conspicuos....... .9

8b. Conidióforos secundarios ausentes o inconspicuos... .10

9a. Cadenas de hasta 5 conidios, ovoides o elipsoides, homogéneos en longitud (18-34 $\mu \mathrm{m})$...... A. daucicaulis

9b. Cadenas de hasta 10 conidios, obclavado o elipsoides a ovales, de longitud variable $(10-57 \mu m)$....A. infectoria

10a. Cadenas de hasta 7 conidios, ovoides $11-53 \times 7-15 \mu \mathrm{m}$. A. alternata

10b. Cadenas de hasta 12 conidios, ovoides o elipsoides 10-60 x 7-29 $\mu \mathrm{m}$. A. brassicicola

11a. Conidios solitarios, esferoides. A. radicina

11b. Cadenas de hasta 3 conidios elipsoides, ocasionalmente solitarios...... A. geophila

11c. Grupos de hasta 3 conidios ovoides o subcilíndricos, ocasionalmente solitarios A. broccoli-italicae 
Tabla 1. Origen de los aislados usados en el estudio

\begin{tabular}{ccccc}
\hline Id. Aislado & Especie & Hospedero & Lugar & Grupo* \\
\hline 25 & A. alstroemeriae & Alstroemeria sp. & Funza & $\mathrm{G}$ \\
\hline 26 & A. alstroemeriae & Gerbera sp. & Funza & $\mathrm{G}$ \\
\hline 27 & A. alstroemeriae & Dahlia sp. & Funza & $\mathrm{G}$ \\
\hline 9 & A. alstroemeriae & Alstroemeria sp. & Bogotá (Suba) & $\mathrm{G}$ \\
\hline 18 & A. alstroemeriae & Alstroemeria sp. & Bojacá & $\mathrm{G}$ \\
\hline 10 & A. alternata & Mangifera indica & Chía & $\mathrm{J}$ \\
\hline 24 & A. alternata & Alstroemeria sp. & Cajicá & $\mathrm{J}$ \\
\hline $31,32,33,34$ & A. alternata & Solanum lycopersicum & Bogotá (Suba) & $\mathrm{J}$ \\
\hline 37 & A. alternata & S. lycopersicum & Mosquera & $\mathrm{J}$ \\
\hline 16 & A. astragali & Prumnopitys sp. & Bogotá (JBJCM**) & $\mathrm{G}$ \\
\hline 28,29 & A. brassicicola & Brassica olearacea & Madrid & $\mathrm{J}$ \\
\hline $41,43,44$ & A. brassicicola & Brassica sp. & Cajicá & $\mathrm{J}$ \\
\hline 47,48 & A. brassicicola & Raphanus sativus & Cota & $\mathrm{J}$ \\
\hline 39,40 & A. broccoli-italicae & B. olearacea & Funza & $\mathrm{G}$ \\
\hline 42 & A. broccoli-italicae & Brassica sp. & Cajicá & $\mathrm{G}$ \\
\hline 15 & A. colombiana & Physalis peruviana & Facatativá & $\mathrm{G}$ \\
\hline 11 & A. daucicaulis & Chenopodium quinoa & Zipaquirá & $\mathrm{K}$ \\
\hline
\end{tabular}

por obscurecimiento de fotografías. Adicionalmente se registró la longitud de los conidióforos.

\section{Características de los conidios}

Los conidios de cada uno de los aislados fueron montados en láminas portaobjetos usando cinta adhesiva y como líquido de montaje agua y azul de lactofenol. Se seleccionaron 500 conidios maduros decada uno de los aislados y se determinó su longitud y ancho, con los cuales se calculó la relación largo-ancho, o el área visible de los conidios, y el número de septos transversales y longitudinales. Se calculó el promedio $(\bar{x})$ y la desviación (s) y el rango (R) de los valores obtenidos (Tabla 2). Además, otras características morfológicas de los conidios fueron consideradas para su descripción, como la forma del pico y la ornamentación de sus paredes. Las características de los conidios fueron registradas fotográficamente a 400X y 1000X de magnificación. 


\begin{tabular}{|c|c|c|c|c|}
\hline 14 & A. gaisen & P. peruviana & Cajicá & G \\
\hline 23 & A. gaisen & Zantedeschia aethiopica & Cajicá & G \\
\hline 6 & A. gaisen & Orchidaceae & Chía & G \\
\hline 2 & A. geophila & Coriandrum sativum & Mosquera & $\mathrm{F}$ \\
\hline 30 & A. geophila & P. peruviana & Bogotá (Guaymaral) & $\mathrm{F}$ \\
\hline 13 & A. godetiae & P. peruviana & Cajicá & G \\
\hline 35 & A. pellucida & S. lycopersicum & Bogotá (Suba) & G \\
\hline $\begin{array}{c}8 \mathrm{a}, 8 \mathrm{~b}, 8 \mathrm{c} \\
8 \mathrm{~d}, 8 \mathrm{e}, 8 \mathrm{f}, 8 \mathrm{~g}\end{array}$ & A. pellucida & Z. aethiopica & Facatativá & G \\
\hline 12 & A. radicina & C. quinoa & Zipaquirá & $\mathrm{A} 1$ \\
\hline 36 & A. tenuissima & S. lycopersicum & Bogotá (Suba) & $\mathrm{H}$ \\
\hline 7 & A. tenuissima & Orchidaceae & Chía & $\mathrm{H}$ \\
\hline 38 & A. infectoria & C. sativum & Mosquera & K \\
\hline $19,20,21,22$ & Curvularia sp. & S. lycopersicum & Cajicá & \\
\hline 1 & Embellisia sp. & S. lycopersicum & El Rosal & \\
\hline 17 & Ulocladium sp. & Rosmarinus officinalis & Cota & \\
\hline 5 & Ulocladium oudemansii & Cucurbitaceae & Chía & \\
\hline
\end{tabular}

* Agrupación por patrón de esporulación de acuerdo a Simmons (2007). ** Jardín Botánico José Celestino Mutis

\section{Descripción y claves taxonómicas}

Las características de las colonias, de los conidios y el patrón de esporulación de cada una de las especies encontradas en el estudio, fueron condensadas en descripciones cortas, acompañadas de fotografías de las características microscópicas. De la misma manera, se presenta una clave taxonómica para la identificación de especies de conidios pequeños en la Sabana de Bogotá. La validación de nuestra clave se realizó comparando contra la propuesta por Simmons (2007).

\section{RESULTADOS Y DISCUSIÓN}

Una colección de 43 aislados de Alternaria, y siete de géneros asociados (Tabla 1), fue obtenida de 13 zonas de la sabana de Bogotá. Con base en sus características morfológicas y en las descripciones realizadas por Simmons (2007), se pudo establecer que los aislados de Alternaria corresponden a 14 especies, pertenecientes en todos los casos a la sección de conidios pequeños. Las especies 


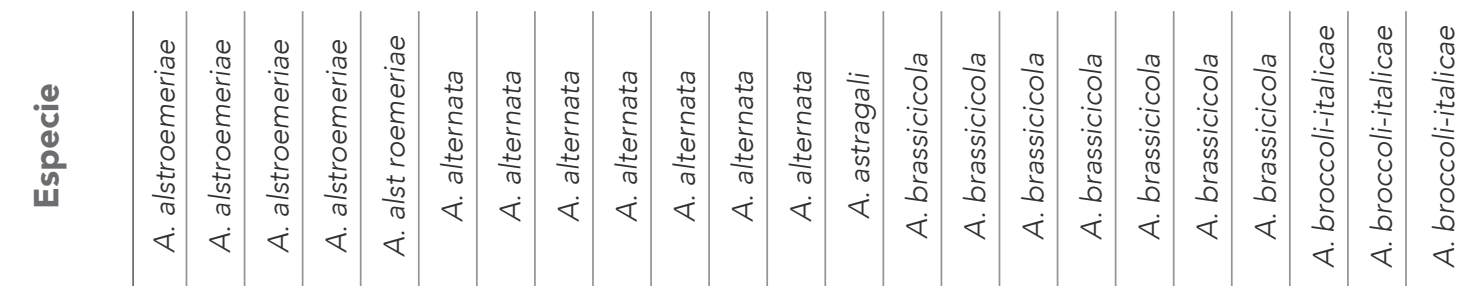

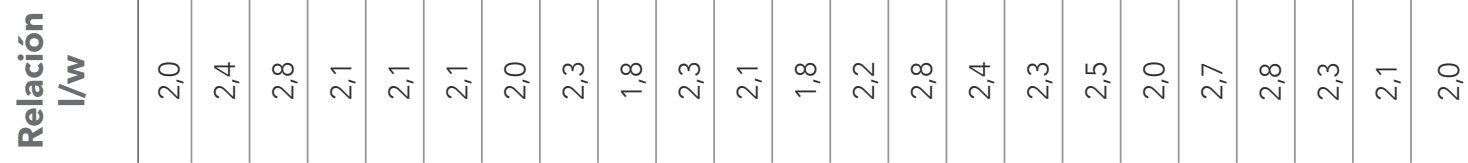

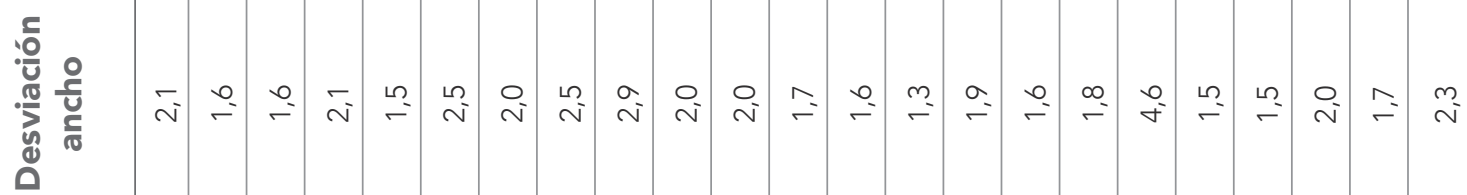

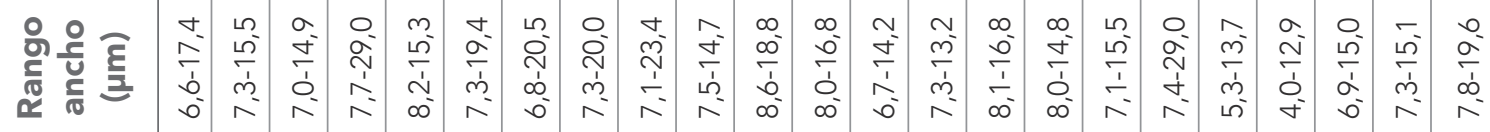

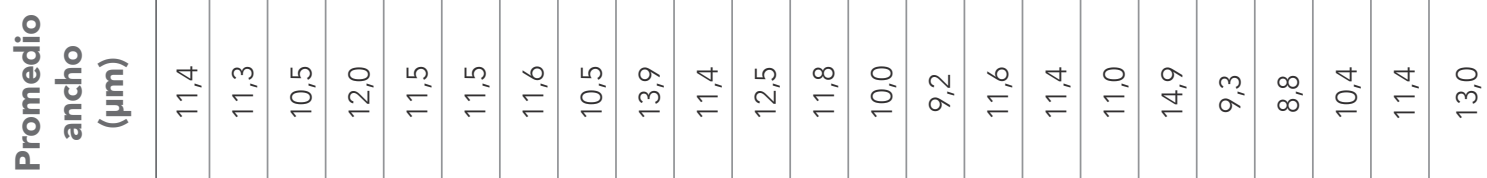

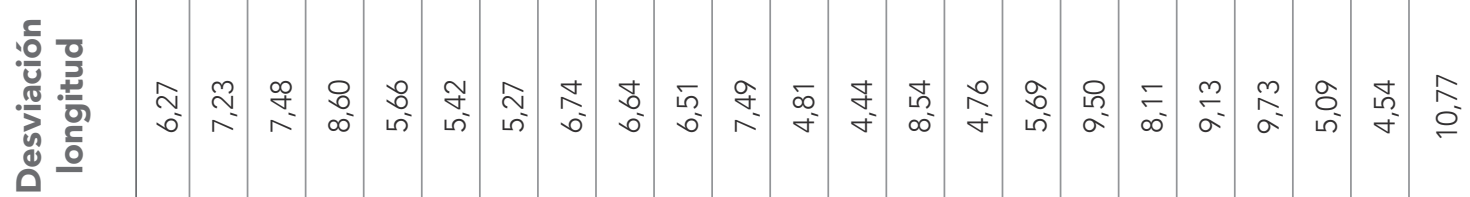

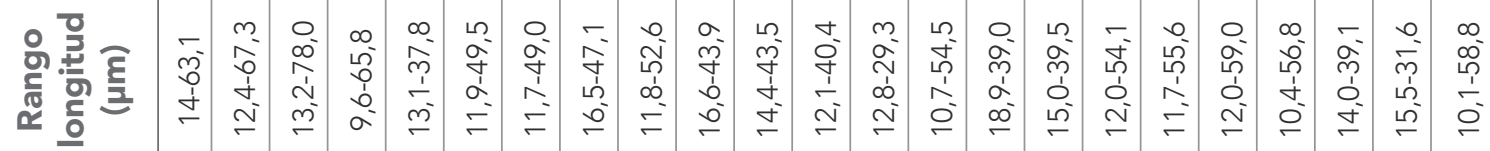

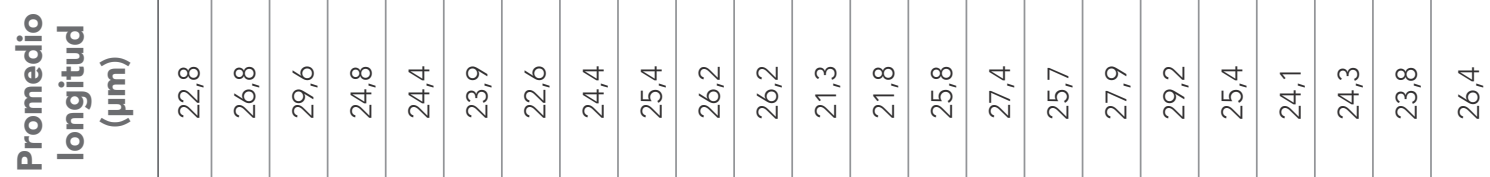

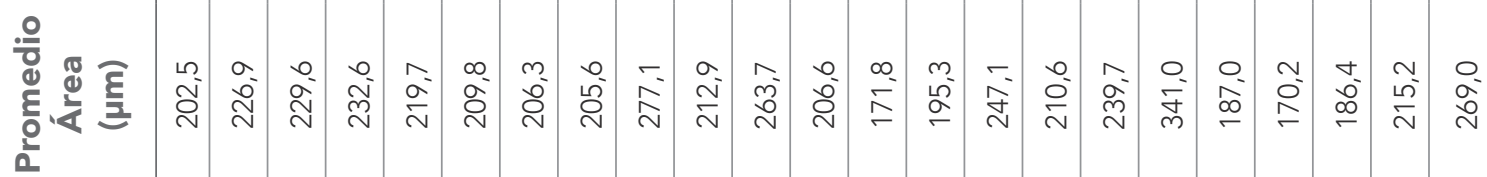

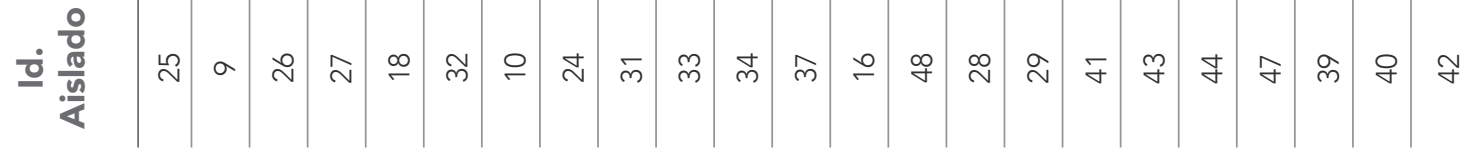




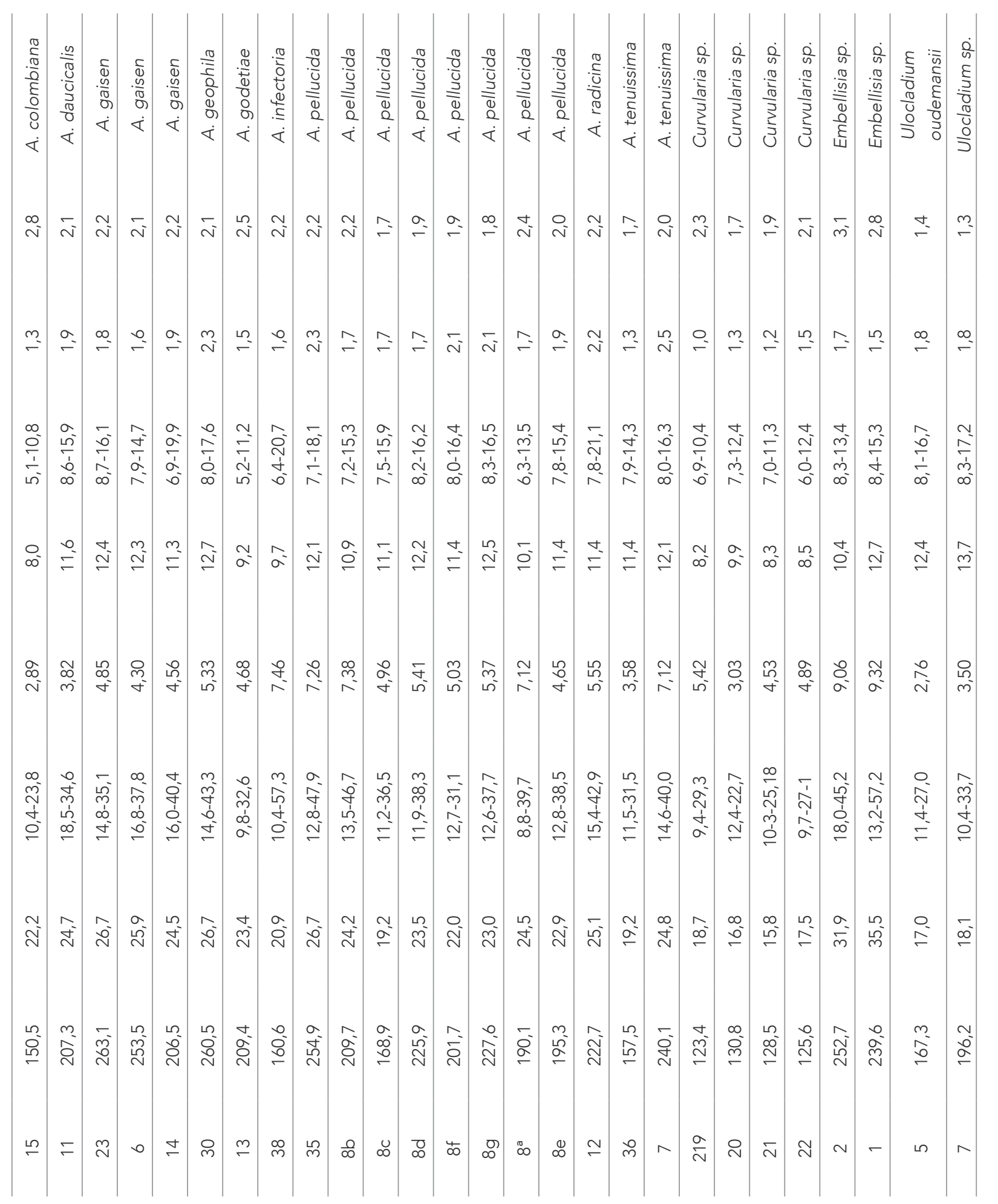




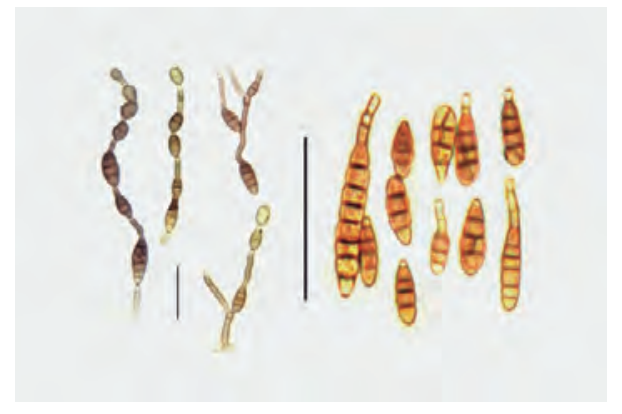

Figura 1. Patrón de esporulación y conidios en A. alstroemeriae, Barra $=50 \mu \mathrm{m}$.

pueden distribuirse en seis grupos (designados con una letra de acuerdo a las claves de Simmons, 2007). El grupo G fue el más abundante e incluye 22 aislados y siete especies, seguido por el grupo J con catorce aislados y dos especies, y el grupo K con dos aislados y dos especies. Los tres restantes grupos están representados por una única especie (Tabla 1).

\section{Descripción de las especies encontradas}

Alternaria alstroemeriae E. G. Simmons \& C. F. Hill

Conidios en cadenas cortas de 3-5 unidades, con ocasionales ramas laterales. Conidióforos secundarios

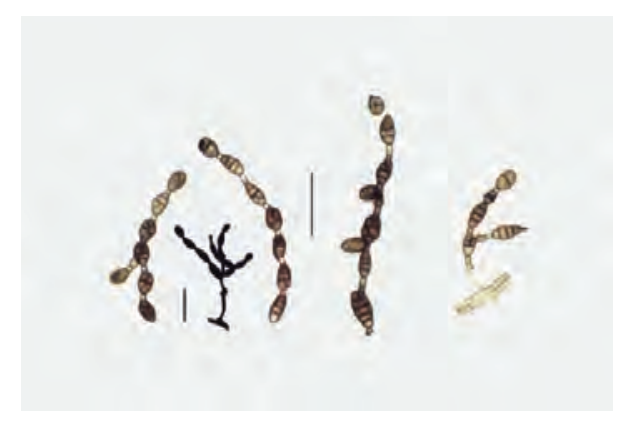

Figura 2. Patrón de esporulación y conidios en A. alternata, Barra $=50 \mu \mathrm{m}$

conspicuos, en algunas ocasiones más largos que la longitud de un conidio. Conidios obclavados, ovoides o subcilíndricos, de $10-80 \times 8-15 \mu \mathrm{m}$, con 2 a 7 segmentos transversales y 0 a 2 segmentos longitudinales. Algunos de los conidios presentan superficie rugosa y color marrón oscuro. Buena parte de la esporulación aparece sumergida en el sustrato (agar). Colonia de 40 $\mathrm{mm}$ de diámetro, de color marrón a verde oliváceo.

\section{Alternaria alternata (Fries) Keissler, Beih}

Conidios en cadenas ramificadas, que se originan cerca del ápice del conidióforo primario. Conidióforos secundarios frecuentemente ausentes. Cadenas de 5 a 7

Las características de las colonias, de los conidios y el patrón de esporulación de cada una de las especies encontradas en el estudio, fueron condensadas en descripciones cortas, acompañadas de fotografías de las características microscópicas. 


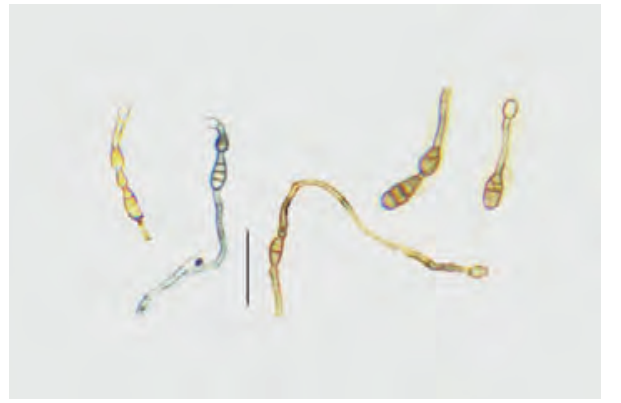

Figura 3. Patrón de esporulación y conidios en A. alternata, Barra $=50 \mu \mathrm{m}$

conidios elipsoides, obclavadosu ovoides de 11-53×7-15 $\mu \mathrm{m}$, con 2-6 septos transversales y 0-3 septos longitudinales. Colonias oscuras verdes o grises, de $45 \mathrm{~mm}$ de diámetro, frecuentemente con varios anillos concéntricos.

Alternaria astragali Wangeline \& E. G. Simmons

Conidióforos primarios largos y erectos que producen cadenas simples de conidios que generan conidióforos secundarios largos. Cadenas con 3-7 conidios. Ramificaciones ausentes. Conidios ovoides o comúnmente largo elipsoides, los conidios apicales aparecen casi esferoides. Conidios de 13$30 \times 7-14 \mu \mathrm{m}$, con $2-5$ septos transversales, septos

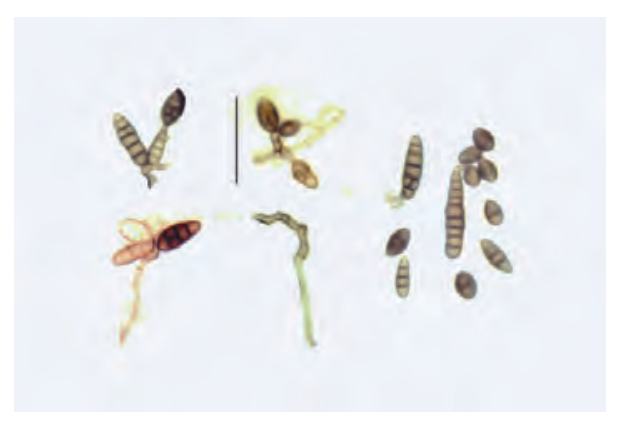

Figura 5. Patrón de esporulación conidióforo y conidios en A. broccoli italicae, Barra $=50 \mu \mathrm{m}$

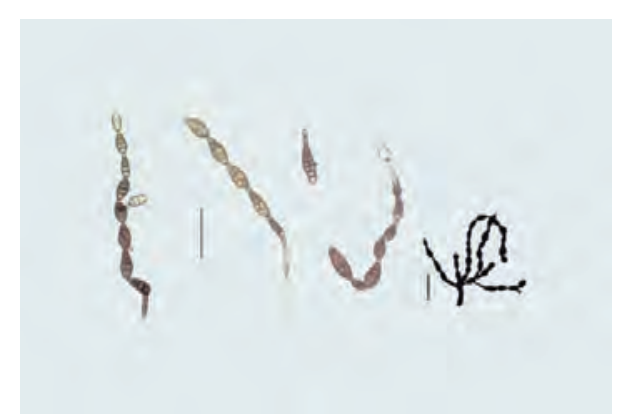

Figura 4. Patrón de esporulación y conidios en A. brassicicola, Barra $=50 \mu \mathrm{m}$

longitudinales, ocasionalmente uno. Colonias verde oliva a marrón oscuro, de $65 \mathrm{~mm}$ de diámetro.

Alternaria brassicicola (Schw.) Wiltshire

Conidióforos primarios cortos que originan cadenas ramificadas de entre 5 y 15 conidios. Conidióforos secundarios cortos o ausentes. Conidios ovoides a elipsoides de 10-60 x 7-29 $\mu \mathrm{m}$. Los conidios presentan de 2 a 7 septos transversales y 0 a 2 septos longitudinales. Colonias de color verde oscuro en la zona central, verde oliva en la parte media (fuertemente esporulada), y con un halo blanco en el borde, donde solo aparecen hifas, de $45 \mathrm{~mm}$ de diámetro.

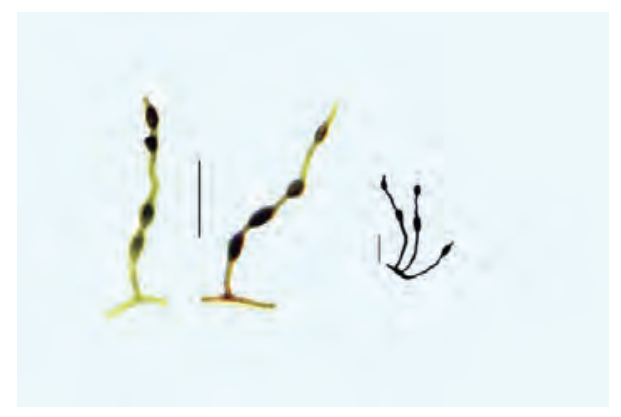

Figura 6. Patrón de esporulación y conidios en A. colombiana, Barra $=50 \mu \mathrm{m}$ 
Alternaria broccoli-italicae E. G. Simmons

Conidióforos primarios cortos, en algunas ocasiones más largos que los conidios y fuertemente retorcidos. Conidióforos secundarios ausentes. Conidios solitarios, en grupos de hasta 3 conidios. Conidios variados, esferoides a elipsoides, los pequeños de 10-25 x 7-10 $\mu \mathrm{m}$, y subcilíndricos los grandes 30-55 x 10-15 $\mu \mathrm{m}$, con el ápice redondeado. Septos longitudinales ausentes o 1 en los conidios grandes, de 2 a 8 septos transversales, dependiendo del tamaño del conidio. Colonias verde oliva hasta casi negras, de $45 \mathrm{~mm}$ de diámetro.

\section{Alternaria colombiana E. G. Simmons}

Conidióforos primarios erectos, conspicuos, de los cuales se originan cadenas lineales de 3-5 conidios, separados por los picos largos filamentosos de los conidios. En algunas ocasiones se generan conidióforos secundarios sobre el conidio apical Conidios generalmente apretado ovoides o elipsoides, de 10 24 × 5-10 $\mu \mathrm{m}$, de 4 a 7 septos transversales y 1 o 2 septos longitudinales. Colonias de $70 \mathrm{~mm}$ de diámetro.

\section{Alternaria daucicaulis E. G. Simmons}

Conidióforos primarios cortos, erectos. Conidióforos secundarios conspicuos y determinantes en el

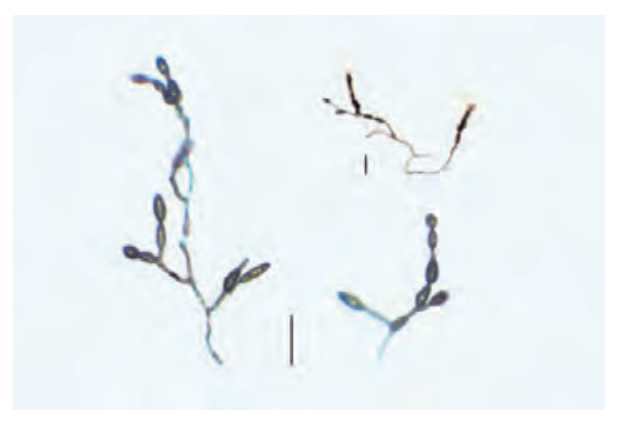

Figura 7. Patrón de esporulación y conidios en A. alternata, Barra $=50 \mu \mathrm{m}$ patrón de esporulación. Pocas ramificaciones, pero siempre presentes. Cadenas de 3-5 conidios, frecuentemente elipsoides, algunas veces ovoides, con una célula apical hialina y roma. Conidios de 20-35 x 9-16 $\mu \mathrm{m}$, con 3-4 septos transversales y 0-2 septos longitudinales. Colonias de $55 \mathrm{~mm}$ de diámetro, con 3 anillos oscuros concéntricos bien marcados.

\section{Alternaria gaisen Nagano}

Conidióforos primarios cortos. Conidióforos secundarios ausentes, cadenas no ramificadas de 5-10 conidios, obclavados, ovoides, pero más frecuentemente ancho-elipsoides, carentes de pico. Conidios de 15-40 x 7-20 $\mu \mathrm{m}$, con 3-5 septos transversales y 0-3 septos longitudinales. Colonias de $60 \mathrm{~mm}$ de diámetro, con varios anillos oscuros concéntricos.

\section{Alternaria geophila Daszewska, Bull}

Conidióforos primarios cortos que soportan 1 conidio, o cadenas cortas de hasta 4 conidios. Ramificaciones cortas. Conidióforos secundarios ausentes. Conidios de 14-43 x 8-18 $\mu \mathrm{m}$, ovoides a elipsoides. De 2 a 6 septos transversales, sin septos longitudinales, ocasionalmente 1. Colonias claras con una densa capa de micelio, con poca

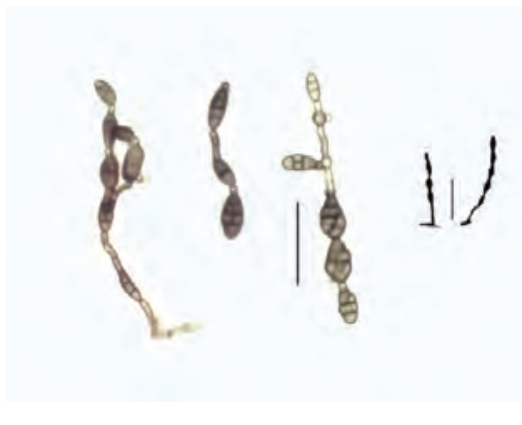

Figura 8. Patrón de esporulación y conidios en A. gaisen, Barra $=50 \mu \mathrm{m}$ 


\section{El género Alternaria posee una gran diversidad morfológica que} ha hecho difícil su taxonomía. Sin embargo, conocer mejor el patógeno permite explorar caminos diferentes en términos de su control, de otra manera se mantienen medidas de control y manejo generales que puden desembocar en uso de estrategias que generan problemas de contaminación, o emergencia de resistencias indeseables.

esporulación, algunos anillos concéntricos un poco más oscuros, de $60 \mathrm{~mm}$ de diámetro.

\section{Alternaria godetiae (Neerg.) Neerg.}

Conidióforos primarios conspicuos que producen cadenas de hasta 6 conidios, no ramificadas. Conidios de 10-30 x 5-10 $\mu \mathrm{m}$, elipsoides o estrecho-ovoides. Conidios con 3-7 septos transversales y sin septos longitudinales, ocasionalmente 1 o 2 . Conidióforos secundarios ausentes. Colonias de $60 \mathrm{~mm}$ de diámetro, con varios anillos concéntricos bien definidos.

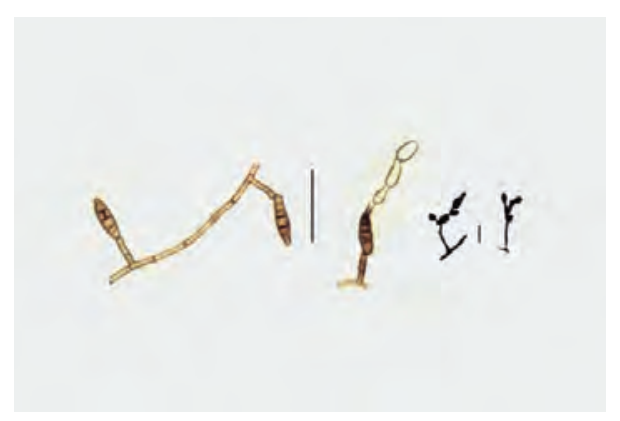

Figura 9. Patrón de esporulación y conidios en A. geophila, Barra = $50 \mu \mathrm{m}$

\section{Alternaria infectoria E. G. Simmons}

Conidióforos primarios conspicuos, frecuentemente más largos que la longitud de un conidio, dan origen a 1 o 2 conidios que forman cadenas lineales de hasta diez unidades, frecuentemente más cortas y ramificadas. Conidióforos secundarios ocasionalmente presentes y de variada longitud. Conidios alargados, apretado-ovoides o estrecho-elipsoides, de 10-57 x 6-20 $\mu \mathrm{m}$. Dependiendo del tamaño del conidio aparecen de 3 a 8 septos transversales y 0 a 3 septos longitudinales. Colonias de $55 \mathrm{~mm}$ de diámetro con una densa capa de

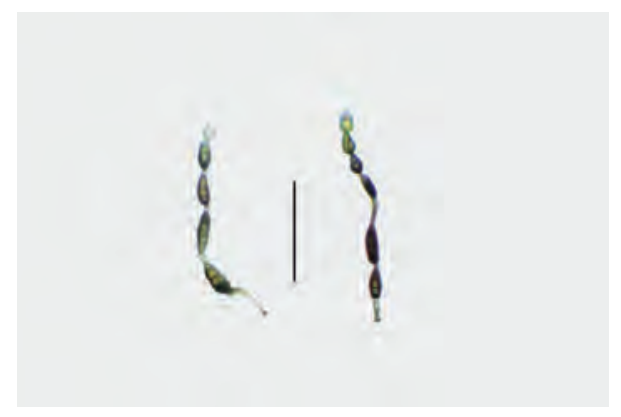

Figura 10. Patrón de esporulación y conidios en A. godetiae, Barra $=50 \mu \mathrm{m}$ 


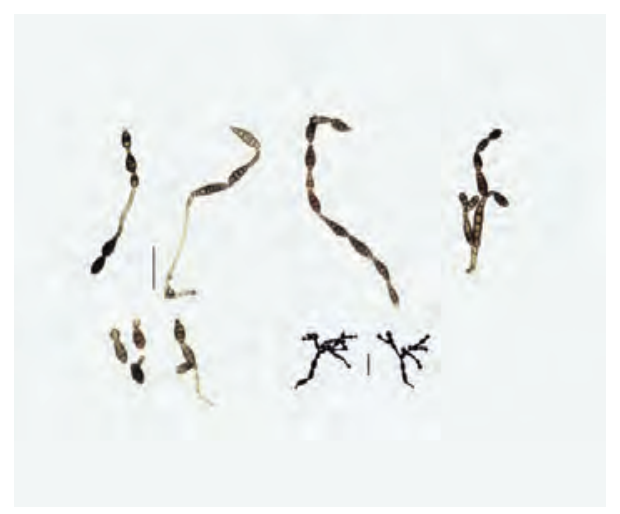

Figura 11. Patrón de esporulación y conidios en A. infectoria, Barra $=50 \mu \mathrm{m}$

micelio, la esporulación es pobre y se concentra en dos anillos concéntricos más oscuros.

\section{Alternaria pellucida E. G. Simmons}

Conidióforos primarios cortos, erectos. Conidióforos secundarios ausentes. Cadenas no ramificadas de 5- 12 conidios. Conidios ovoides, frecuentemente ancho elipsoide. Conidios de 11-50 x 7-18 $\mu \mathrm{m}$, presentan 3-6 septos transversales y 1-2 septos longitudinales. Algunos conidios con la pared rugosa. Colonias de $75 \mathrm{~mm}$ de diámetro, con varios anillos concéntricos.

\section{Alternaria radicina Meier, Drechsler \& Eddy}

Conidióforos primarios erectos, que pueden ramificarse y originan grupos de conidios en los ápices Conidios variados, algunos, relativamente largos, elipsoides, largo elipsoides, hasta subcilíndricos, con 5 a 7 septos transversales y 1 ○ 2 septos longitudinales, otros cortos, ovoides obovoides a casi esferoides, con

Por último, en trabajo se muestra como, a través del uso de la información morfológica, se puede generar una clave de uso regional para identificar las especies de este hongo que esperamos sea de utilidad entre los responsables de laboratorios de diagnóstico en la Sabana de Bogotá. 


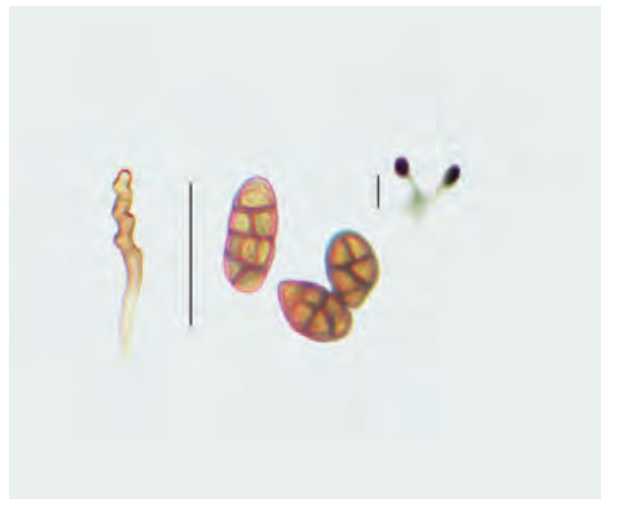

Figura 13. Patrón de esporulación, conidióforo y conidios en A. radicina, Barra $=50 \mu \mathrm{m}$

2 a 4 septos transversales y 1 o 2 longitudinales. Conidios largos 25-45 x 10-20 $\mu \mathrm{m}$, conidios cortos 15-25 x 7-15 $\mu \mathrm{m}$. Algunos de los conidios presentan la pared rugosa. Colonias de $40 \mathrm{~mm}$ de diámetro.

\section{Alternaria tenuissima (Ness \& T. Ness : Fr.)}

\section{Wiltshire}

Conidióforos primarios cortos. Cadenas de 5-15 conidios, algunas veces con una ramificación corta, originada en los conidios. Conidios ovoides o elipsoides, frecuentemente con 4 septos transversales y 1 longitudinal, conidios maduros más grandes, estrecho-ovoides, hasta 7 septos longitudinales y 3 transversales. Algunos conidios presentan un picocónico de 2 a 3 células de largo. Conidios de 11-40 x 8-16 $\mu \mathrm{m}$, algunas veces con la pared rugosa. Colonias de $70 \mathrm{~mm}$ de diámetro.

\section{CONCLUSIONES}

El género Alternaria posee una gran diversidad morfológica que ha hecho difícil su taxonomía. Sin embargo, conocer mejor el patógeno permite

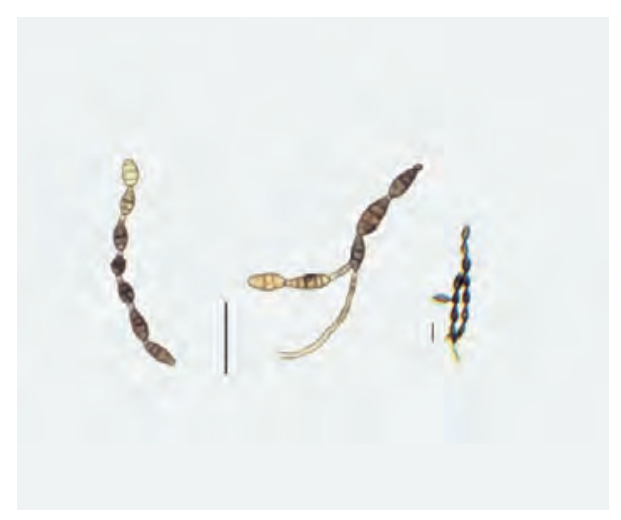

Figura 14. Patrón de esporulación y conidios en A. tenuissima, Barra $=50 \mu \mathrm{m}$

explorar caminos diferentes en términos de su control, de otra manera se mantienen medidas de control y manejo generales que puden desembocar en uso de estrategias que generan problemas de contaminación, o emergencia de resistencias indeseables. Por otra parte, el poder hacer una determinación de las especies de un género en una zona geográfica, nos acerca al conocimiento de la diversidad en esa zona. Por último, en trabajo se muestra como, a través del uso de la información morfológica, se puede generar una clave de uso regional para identificar las especies de este hongo que esperamos sea de utilidad entre los responsables de laboratorios de diagnóstico en la Sabana de Bogotá.

\section{AGRADECIMIENTOS}

Agradecemos a la Dra. Silvia Restrepo la lectura crítica de este trabajo y sus observaciones acertadas. Este trabajo fue financiado por la Vicerrectoría de Investigaciones de la Universidad Militar Nueva Granada, proyecto CIAS-940, a la cual agradecemos su apoyo financiero. 


\section{BIBLIOGRAFÍA}

1. Aly, A., Edrada-Ebel, R., Indriani, I., Wray, V., Müller, F., Totzke, F., Zirrgiebel, U., Schächtele, C., Kubbutat, M., Lin, W., Proksch, P., Ebel, R. 2008. Cytotoxic Metabolites from the Fungal Endophyte Alternaria sp. and Their Subsequent Detection in Its Host Plant Polygonum senegalense. Journal of Natural Products 71:972-980

2. Agrios GN. 2005. Plant Pathology. $5^{\text {th }}$. Ed. Elservier Academic Publisher, California, 922 p.

3. Andersen, B., Kroger, E., Roberts, R. 2001. Chemical and morphological segregation of Alternaria alternata, A. gaisen, and A. longipes. Mycological Research 105:291-299.

4. Andrew, M., Peever, T., Pryor, B. 2009. An expanded multilocus phylogeny does not resolve morphological species within the small-spored Alternaria species complex. Mycologia 101:95-109.

5. Barkai-Golan, R. 2008. Alternaria mycotoxins. P. 185-203. En: Barkai-Golan, R. Nachman P (Eds.). Mycotoxins in fruits and vegetables. Ed. Academic Press, San Diego, 395 p.
6. Barreto, J., Aguirre, M., Caicedo, A., Miranda, D., Echeverri, L., Campos, Y. 2002. El cultivo del tomate tipo milano, pimentón, maíz dulce y fríjol en el sistema de siembra de camas plastificadas bajo las condiciones agroecológicas de la meseta de Ibagué. Editorial El Poira, Ibagué, 49 p.

7. Castro, B., Timmer, L., Muller, G. 2000. Enfermedades de los cítricos en Colombia. Bogotá, Produmedios, $101 \mathrm{p}$.

8. Chou, W., Wu, W. 2002. Phylogenetic analysis of internal transcribed spacer regions of the genus Alternaria, and the significance of filament-beaked conidia. Mycological Research, 106:164-169.

9. Cline, E., Farr, D., Rossman, A., Palm, M., McCray, E. Fungal Nomenclature Database, Systematic Mycology and Microbiology Laboratory, ARS, USDA. http://nt.ars-grin.gov / fungaldatabases/nomen/Nomenclature.cfm, consulta Enero de 2014

10. Ellis, M. (1971). Dematiaceous Hyphomycetes. CMI, Kew, Surrey, England, 608 p. 
11. Ellis, M. (1976). More Dematiaceous Hyphomyctes. CMI, Kew. Surrey, England, 507 p.

12. Farr, D., Rossman, A. Fungal Databases, Systematic Mycology and Microbiology Laboratory, ARS, USDA. http://nt.ars-grin.gov/ fungaldatabases/ consulta Julio de 2013

13. Joly, P. 1964. Le Genre Alternaria. Encyclopédie Mycologique, Paul Lechevalier, éditeur. Paris $250 \mathrm{p}$.

14. Kaur, T., Sharma, D., Kaur, A., Manhas, R. 2013. Antagonistic and plant growth promoting activities of endophytic and soil actinomycetes. Archives Of Phytopathology And Plant Protection 46:1756-1768.

15. Kirk, P., Cannon, P., Minter, D., Stalpers, J. 2008. Ainsworth and Bisby's Dictionary of the Fungi. 10th ed. CABI International. Wallingford, UK, $771 \mathrm{p}$.

16. Lahlali, R., Hijri, M. 2010. Screening, identification and evaluation of potential biocontrol fungal endophytes against Rhizoctonia solani AG3 on potato plants. FEMS Microbiology Letters 311:152-159.
17. Lantigua, P., Martinez, C., Pons-Kühnemann, J., Pérez, M. 2008. Grupos intraespecíficos de Alternaria solani de tomate y papa. Fitopatología Venezolana 21:44-50

18. Neergaard, P. 1945. Danish species of Alternaria and Stemphylium. Oxford University Press, Copenhagen, Denmark, 560p.

19. Orjuela, J. 1965. Índice de las enfermedades de las plantas cultivadas en Colombia. Boletín técnico 11. Instituto Colombiano Agropecuario, Bogotá, $66 \mathrm{p}$.

20. Ostry, V. 2008. Alternaria mycotoxins: An overview of chemical characterization, producers, toxicity, analysis and occurrence in foodstuffs. World Mycotoxin 1: 175-188

21. Pitt, J., Hocking, A. 1997. Fungi and food spoilage. $2^{\text {nd }}$ edn. Blackie Academic and Professional, London, 519 p.

22. Pryor, B., Bigelow, D. 2003. Molecular characterization of Embellisia and Nimbya species and their relationship to Alternaria, Ulocladium, and Stemphylium. Mycologia 95:1139-1152. 
23. Pryor, B., Gilbertson, R. 2000. Molecular phylogenetic relationships amongst Alternaria species and related fungi based upon analysis of nuclear ITS and mt SSU rDNA sequences. Mycological Research 104: 1312-1321.

24. Roberts, R., Reymond, S., Andersen, B. 2000. RAPD fragment pattern analysis and morphological segregation of smallspored Alternaria species and species groups. Mycological Research 104:151-160.

25. Rotem, J. 1994. The Genus Alternaria: Biology, Epidemiology and Pathogenicity. APS press, St Paul, MN, USA, $326 \mathrm{p}$.

26. Simmons, E. 1992. Alternaria taxonomy: current status, viewpoint, challenge. p. 1-35. En: Chelkowski J. y Visconti A. (Eds). Alternaria Biology, Plant Diseases and Metabolites. Elsevier Science Publishers, Amsterdam, Netherlands, $573 \mathrm{p}$.

27. Simmons, E. 1993. Alternaria themes and variations (54-62). Mycotaxon 46:171-199.

28. Simmons, E. 1997. Alternaria Themes and Variations (151-223). Mycotaxon 65:1-91.

29. Simmons, E. 1999. Alternaria Themes and Variations (236-243). Host specific toxins producers. Mycotaxon 70:325-369.
30. Simmons, E. 2007. Alternaria. An Identification Manual. CBS Fungal Biodiversity Centre, Utrecht, Netherlands, $780 \mathrm{p}$.

31. Simmons, E, Roberts R. 1993. Alternaria themes and variations (73). Mycotaxon 48:109-140.

32. Sun, X., Zhang, T. 2008. Morphological and molecular characterization of Alternaria isolates on fruits of Pyrus bretschneideri Rehd. "Ya Li". Mycosystema 27:105-117.

33. Woudenberg, J., Groenewald, J., Binder, M., Crous, P. 2013. Alternaria redefined. Studies in Mycology 75:171-212.

34. Zapata, J., Saldarriaga, A., Londoño, M., Díaz, C. 2002. Manejo del cultivo de la uchuva en Colombia. Boletín Técnico 14. Centro de Investigación La Selva, Corpoica. Rionegro, Antioquia, $41 \mathrm{p}$.

35. Zhang, T. 2003. Alternaria, Flora Fungorum Sinicorum. Vol. 16. Science Press, Beijing, 283 p.

36. Zubieta, D, Alarcón P. 1974. Evaluación de las enfermedades del pimentón (Capsicum annum L.) en el Valle del Cauca, Colombia. Trabajo de grado. Facultad de Ciencias Agropecuarias, Universidad Nacional de Colombia, Palmira, $157 \mathrm{p}$. 
Revista ULTAD DE CIENCIAS BÁSICAS 247 